\title{
The CT Band, CT Band Biomechanics and CT Band Syndrome
}

\author{
by Jeffrey Oster, DPM, FACFAS 1
}

The Foot and Ankle Online Journal 2 (5): 2

Walking requires the effective transfer of force between the lower extremity and the supporting surface. This transfer of force is accomplished by a diverse group of muscles and tendons collectively known as the CT band. CT band anatomy, biomechanics and dynamics are introduced in this paper. The pathomechanics of the CT band, called CT band syndrome, is also described.

Keywords: CT band, CT band biomechanics, CT band dynamics. CT band syndrome, CT band index

$\mathrm{P}$ lantarflexion at the ankle is accomplished by eight different muscles and tendons. Collectively, these muscles, tendons, their origins and insertions are called the CT band. The CT band is a lever that transfers vertical force to a horizontal surface. The abbreviation CT describes the span of this anatomical band extending from the calf $(\mathrm{C})$ to the toes $(\mathrm{T})$.

A lever accomplishes work by delivering force from an effort arm, through the lever, to a resistance arm. The CT band performs work by delivering force from the calf to the forefoot. This task is performed over and over again as we walk. Disproportionate, excessive, or repetitive loading of any segment of the CT band will result in injury. These injuries are collectively called CT band syndrome.

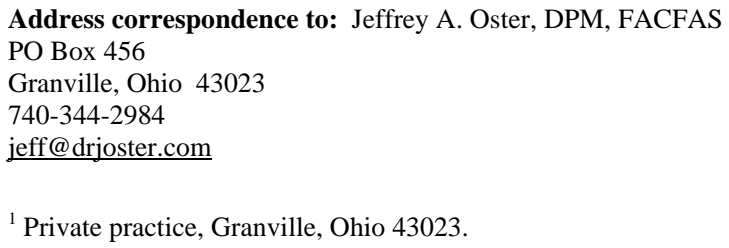

\section{Anatomy of the CT Band}

Levers consist of three components. These components include an effort arm, a fulcrum and a resistance arm. CT band anatomy can be broken down into these three components

\section{The effort arm}

Extrinsic plantarflexors of the foot:

1. The gastrocnemius muscle and Achilles tendon.

2. The soleus muscle and Achilles tendon.

3. The posterior tibial muscle and tendon.

4. The peroneus longus muscle and tendon.

5. The peroneus brevis muscle and tendon.

6. The flexor hallucis longus muscle and tendon.

7. The flexor digitorum longus muscle and tendon.

8. The plantaris muscle and tendon.

Additional effort arm structures:

1. The tibia and fibula

2. The gastrocnemius aponeurosis 


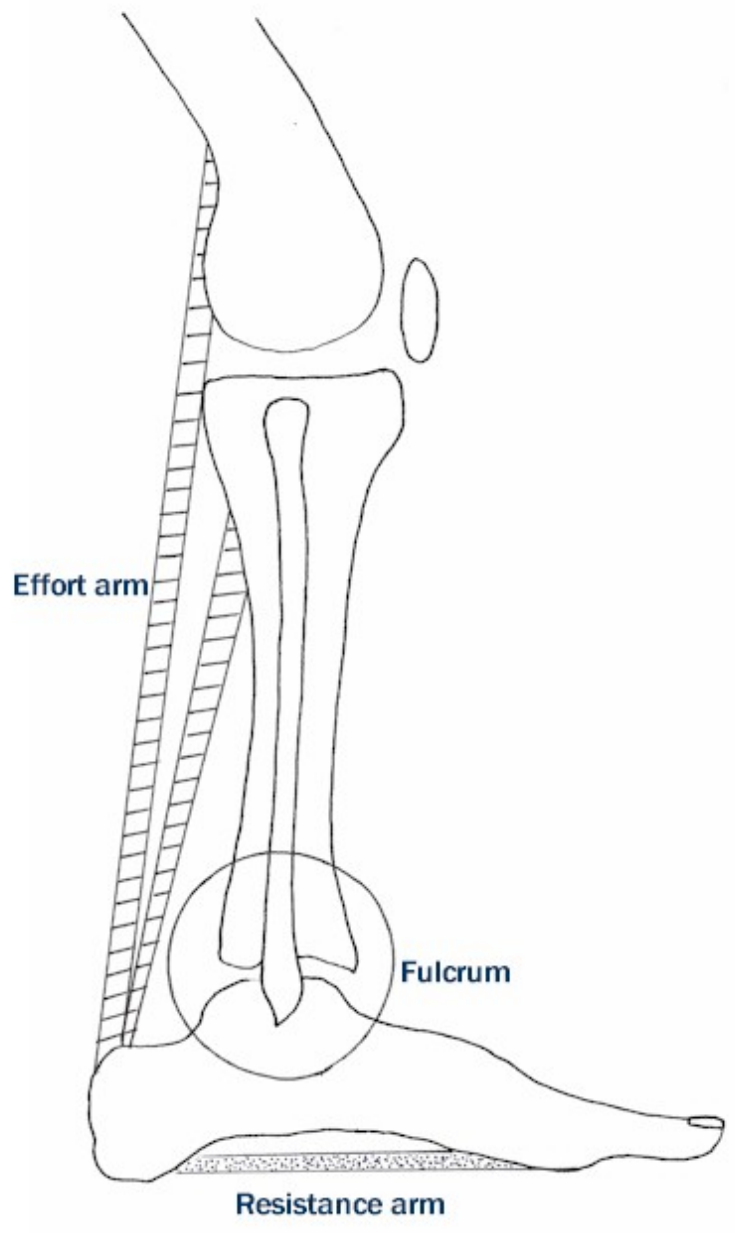

Figure 1 Schematic of the CT band.

\section{The resistance arm}

Intrinsic musculature of the foot:

The abductor hallucis.

The flexor digitorum brevis.

The flexor hallucis brevis.

The abductor digiti minimi.

The quadratus plantae.

The lumbricales and interosseous muscles.
Additional resistance arm structures:

1. Ligaments.

2. Fascia.

3. Bones and joints of the foot.

\section{The fulcrum}

The fulcrum of The CT Band is the joint axis of the talo-crual joint.

\section{CT Band Biomechanics}

CT band biomechanics can be broken into two categories: kinetics and kinematics. Kinetics is the study of motion with respect to force and load. Kinematics is the study of motion without respect to force or load and deals with the fundamental concepts of space, time, velocity and acceleration.

\section{CT Band Kinetics}

\section{Definitions}

\section{Force}

A. Primary force - force generated by the muscles of the CT band.

B. Secondary force - force that originates in a proximal body segment that is transferred distally to the CT band. Secondary force is delivered to the CT band by multi-articular muscles. The CT band transfers secondary force from the hamstrings to the foot. ${ }^{1,2,3}$

\section{Load}

A. Static load - Static load refers to body weight.

B. Non-static load - variable load. Examples of nonstatic load would include a backpack on a hiker, a mother carrying an infant or a worker carrying materials specific to his or her job. 


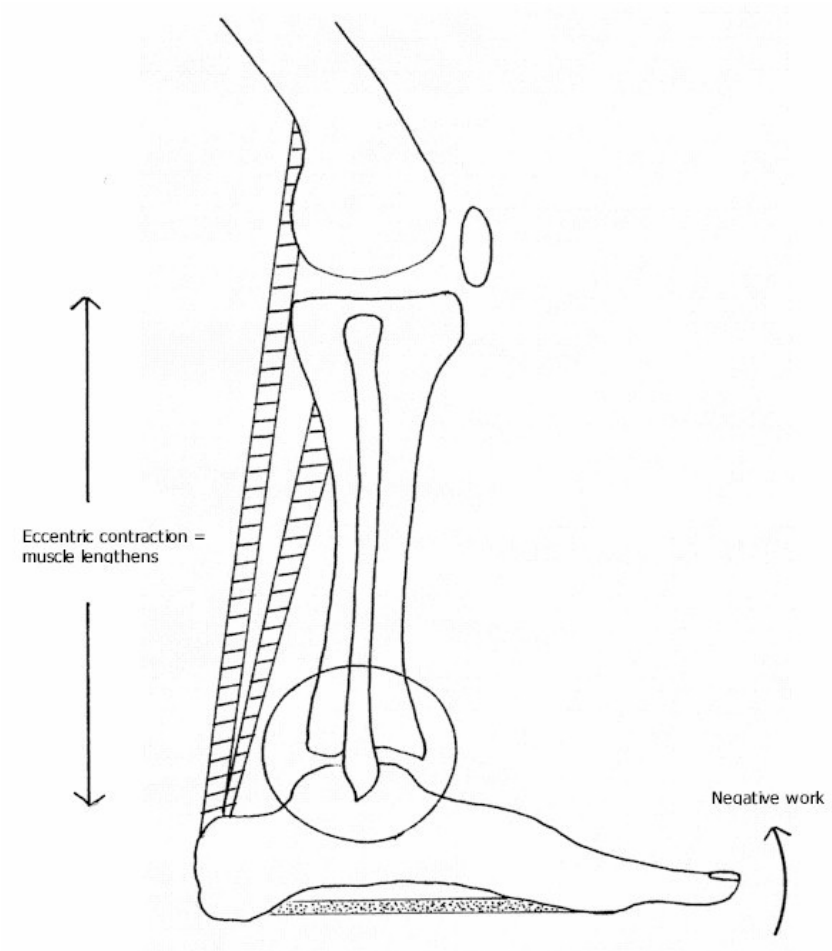

Figure 2 Eccentric muscle contraction.

\section{Eccentric Contraction}

Eccentric contraction occurs when a muscle performs work while lengthening. (Fig. 2) Eccentric contraction has been called negative work, negative contraction, lengthening contraction and decelerator muscle function. ${ }^{4,5}$ Eccentric contraction is the force that is developed by a muscle as it is overcome by an opposing force or load when it can merely provide passive or active resistance. ${ }^{7}$

Figure 2 Eccentric muscle contraction.

Eccentric contraction plays a vital and dominant role in CT band biomechanics. Voluntary eccentric contraction can generate more muscle tension during eccentric muscle group action than what is measured during voluntary concentric or isometric contraction. ${ }^{8}$ More importantly, the amount of work required by

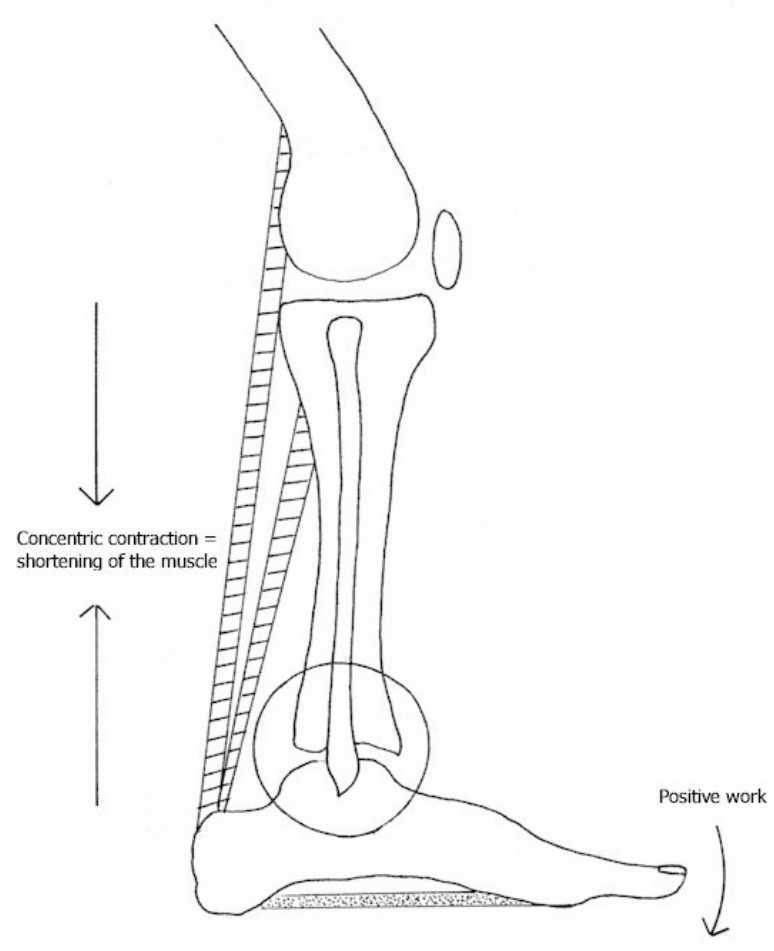

Figure 3 Concentric muscle contraction.

skeletal muscle for eccentric contraction is far less than that required for concentric contraction. ${ }^{\text {? }}$

This finding was confirmed by electromyography (EMG) studies noting that less EMG activity is present for eccentric contraction as compared to concentric contraction lifting the same load. ${ }^{10}$ The CT band takes advantage of this unique, energy saving characteristic of eccentric contraction. It is important to recognize that eccentric rather than concentric contraction dominates CT band biomechanics. 
Table 1. Percentage of Force Contributed By The Individual Muscles of The CT Band.

\begin{tabular}{|l|l|}
\hline Individual Muscle & $\begin{array}{l}\text { Individual muscle percentage as compared to } \\
\text { the group }\end{array}$ \\
\hline Soleus & $55.2 \%$ \\
\hline Gastrocnemius & $37.5 \%$ \\
\hline Tibialis Posterior & $1.0 \%$ \\
\hline Flexor Hallucis Longus & $3.3 \%$ \\
\hline Flexor Digitorum Longus & $1.0 \%$ \\
\hline Peroneus Longus & $1.5 \%$ \\
\hline Peroneus Brevis & $0.5 \%$ \\
\hline & \\
\hline $\begin{array}{l}\text { Adapted from Gait Analysis, Normal and } \\
\text { Pathological Function. (12) }\end{array}$ & $\begin{array}{l}\text { * Percentages were originally defined as the } \\
\text { percentage of the soleal muscle. These figures } \\
\text { have been converted to the percentage of each } \\
\text { individual muscle compared to the entire group. }\end{array}$ \\
\hline
\end{tabular}

\section{Concentric Contraction}

Concentric contraction is also called positive work or positive contraction. (Fig. 3) A concentric contraction produces work as a muscle shortens. CT band concentric contraction occurs during short bursts of activity where quick and abrupt action is required. As previously noted, concentric contraction requires the expenditure of more energy compared to eccentric contraction.

Therefore, in CT band biomechanics, concentric contraction is minimized in an effort to conserve energy.

The anatomical and physiological properties of concentric contraction of the muscles of the CT band have been studied extensively. The majority of concentric force generated by the muscles of the CT band comes from the triceps surae muscle group. ${ }^{6,11-17}$

Additional force is generated by the extrinsic plantar flexors of the lower leg. Table 1 describes the percentage of force contributed by each of the muscles of the CT band.
Table 2 describes the anatomical cross section, lever arm and potential moments of each of the muscles of the CT band.

Tables 1 and 2 show data based exclusively upon concentric muscle contraction of the CT band. Similar testing has not been performed to assess eccentric muscle contraction of the CT band. Until more in-depth studies of eccentric muscle contraction can be performed, we must assume that the properties of both concentric and eccentric contraction are similar. For instance, the subset of data in Tables 1 and 2 would not change when evaluating the same muscle for eccentric vs. concentric contraction. The size of the muscle, the lever arm and even the direction in which the force is being applied to the muscle is the same. The only difference would be the direction in which the muscle is changing: shortening (concentric) or lengthening (eccentric).

The individual kinetic properties of the CT band are difficult to isolate and define in vivo. Static and nonstatic load are the exception in that they can be defined and measured. 
Table 2. Potential Moments of the muscles of the CT Band.

\begin{tabular}{|l|l|l|l|}
\hline Muscle & $\begin{array}{l}\text { Mean anatomical cross } \\
\text { section }(\text { sq. cm.) }\end{array}$ & Lever Arm $(\mathrm{cm})$ & Moment $(\mathrm{kg} . / \mathrm{cm})$. \\
\hline & & & \\
\hline Soleus & 84.1 & 4.9 & 1648 \\
\hline Gastrocnemius & 57.3 & 4.9 & 1123 \\
\hline Tibialis posterior & 16.9 & 2.4 & 162 \\
\hline Peroneus longus & 13.9 & 2.3 & 128 \\
\hline Peroneus brevis & 6.7 & 2.3 & 62 \\
\hline Flexor hallucis longus & 13.4 & 2.4 & 129 \\
\hline $\begin{array}{l}\text { Flexor digitorum } \\
\text { longus }\end{array}$ & 5.5 & 2.4 & 53 \\
\hline
\end{tabular}

(Reprinted from Sutherland DH, An Electromyographic Study Of The Plantar Flexors Of The Ankle In Normal Walking On the Level. (11) Mean anatomical cross sections of the CT Band were published by Weber in 1851. (18) Anatomical cross sections are obtained by dividing the weight of each muscle by its' length. Lever arm determinations, as measured by Jergesen (19) represent the length in the sagital plane between the anatomical axis of the tibia and location of the tendon. Haxton(20) presented the potential moment of each muscle.

Primary and secondary force will vary with activity, rate of gait and variations in types of walking surfaces. Secondary force was estimated by Spoor to be $25 \%$ of the total work performed by the CT band at the level of the ankle. ${ }^{3}$ Eccentric and concentric contraction will also vary in response to situational needs such as rate of gait or changes in walking surfaces. It's important to recognize that the kinetic properties of the CT band are dynamic, responding to situational needs with each and every step.

\section{CT Band Kinematics}

The three kinematic properties of CT band biomechanics are planal dominance, rocker mechanics and rotational placement of the foot.

\section{Planal Dominance}

Levers work most effectively in a single body plane. Linear motion in a single plane is called translation. Deviation of the lever from a single plane results in rotation and a subsequent attempt by the lever to regain single plane function. Figure 4 shows examples of an effective and ineffective lever based upon planal dominance and rotation.
The concepts of translation and rotation in CT band biomechanics are very important. The primary objective of the CT band is to move the body's center of mass forward in the sagital plane with as little energy expenditure as possible. Any deviation from the sagital plane by either the CT band or the body as a whole requires unnecessary work resulting in unnecessary expenditures of energy. Another way to describe rotation within the CT band is to use Newton's first law of motion. Newton stated that if a line of force passes through the center of mass, there will be no rotation on that body. In normal subjects, the CT band moves the body's center of mass forward with less than 5 degrees of deviation from the sagital plane..$^{21,22,23}$

Rotation does occur within the CT band but is typically compensated for by reciprocal or compensatory motion within the lower extremity. For example, transverse plane motion occurs between the leg and supporting surface during gait. The leg moves from an internally rotated position at heel strike to an externally rotated position at the toe off phase of gait. This transverse plane rotation is accomplished proximally by rotation of the pelvis. 

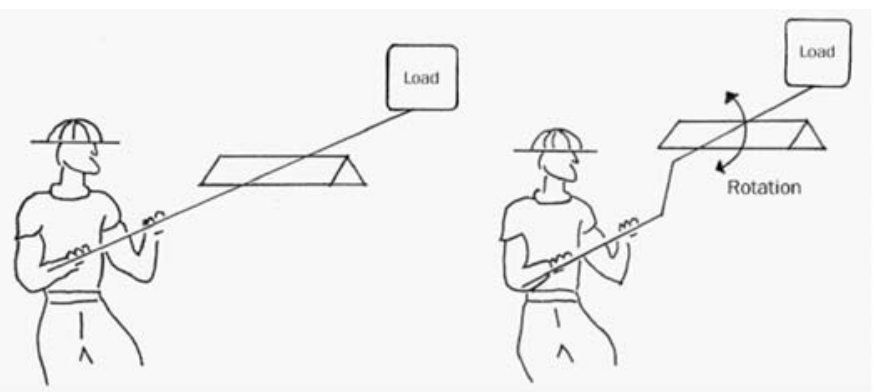

Figure 4 Planal dominance of levers.

Distal compensation is accomplished by motion at the subtalar and midtarsal joints. Although the transverse plane relationship between the leg and the floor changes, the CT band continues to perform with sagital plane dominance throughout gait. This relationship can be described as translation with compensated rotation. Figure 5 shows graphic examples of translation within the sagital plane and rotation in the frontal and transverse planes.

The greater the ability of a lever to function within a single plane, the more effective the lever will be. However a rigid lever is a lever that is unable to absorb strain or adapt to variations in applied load and supporting surfaces. CT band biomechanics requires a degree of rotation to adapt to a constantly changing environment. Rotation is the quality of the CT band that enables the foot and leg to (1) absorb stress as a flexible structure (2) deliver force as a rigid structure and (3) adapt to uneven surfaces.

\section{CT Band Rockers}

A rocker is a mechanical tool that improves the efficiency of the transfer of force and load. The CT band is extremely effective in using three serial rockers: the heel, the ankle and the forefoot. The role of the CT band rockers is two fold. First, rocker mechanics are used to limit the excursion of the body's center of mass. ${ }^{4}$ The second function is to limit the forward motion of the tibia. ${ }^{11,16,25,26}$

Sutherland was the first to describe the unique function of rockers in the foot and ankle. ${ }^{11}$ Figure 6 A shows the excursion that the knee would incur without a foot. Figure $6 \mathrm{~B}$ shows the excursion of the knee without and ankle.

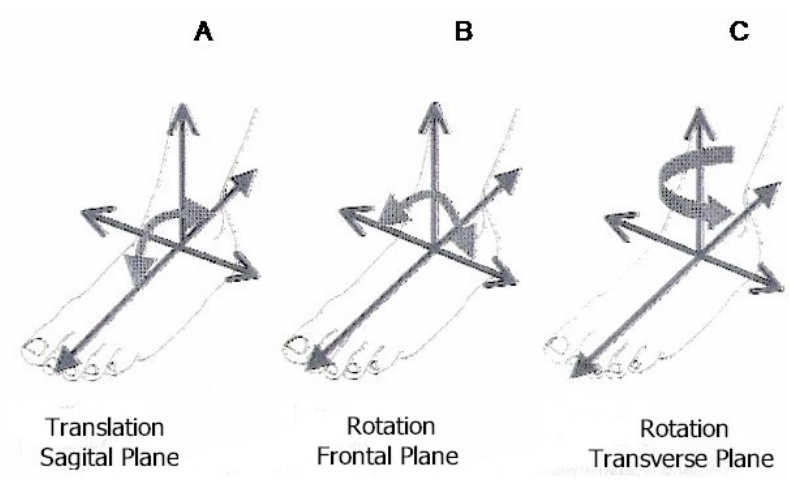

Figure 5 Shows translation in the sagital plane (A). Deviation from the sagital plane, or rotation, is described as inversion/eversion (B) or internal/external rotation (C). (Reprinted with permission from Cornwall MW, McPoil TG. Reliability and validity of center of pressure quantification. ${ }^{24}$
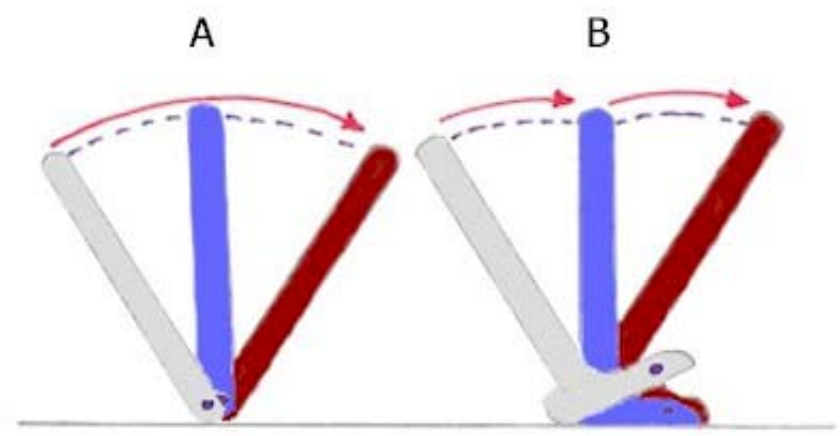

Figure 6 Showing rocker function $(A)$ without a foot and (B) without an ankle.

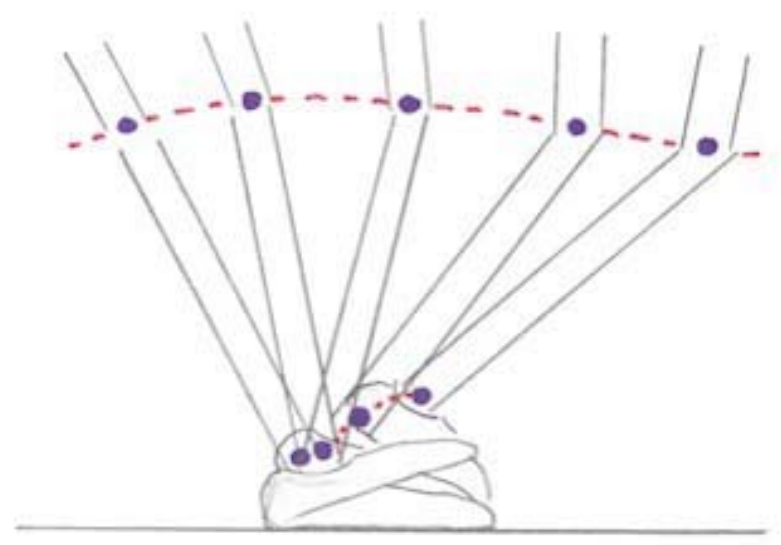

Figure 7 Showing normal rocker function. 


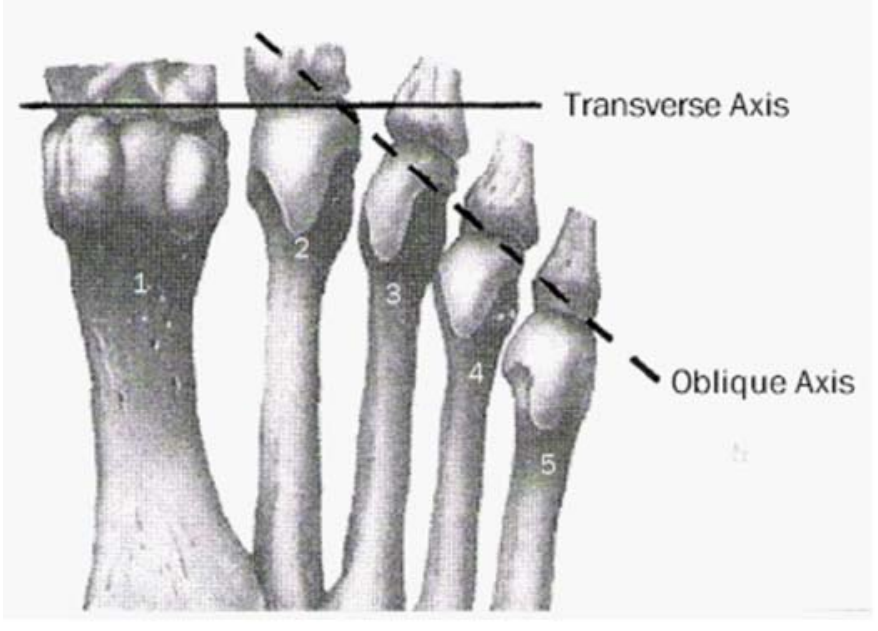

Figure 8 Transverse and oblique axis of the forefoot. Reprinted with permission from Gray's Anatomy, W.B. Saunders Company.

The combined effect of all three rockers of the foot and ankle are better exemplified in Figure 7.

Multiple, serial rockers are an effective way to transfer mechanical force and load while minimizing the body's expenditure of energy. The CT band uses three rockers in a series to effectively move the body's center of mass forward over the foot. The heel rocker and ankle rocker initiate translation at heel contact. Heel rocker translation ends at the beginning of the midstance stage of gait. Ankle rocker function continues, constantly maintaining a position related to, and usually beneath the body's center of mass. The ankle rocker continues forward progression until 10 degrees of dorsiflexion is achieved at the ankle. As the heel-off phase of gait begins, the ankle rocker transfers motion to the forefoot rocker. The forefoot rocker continues translation until the toe-off phase of gait is complete.

\section{Rotational Placement of the Foot}

Rotational placement of the foot has a profound effect on CT band biomechanics by lengthening and shortening the length of the resistance arm of the CT band. ${ }^{27}$ Abduction and adduction of the foot in the transverse plane results in two distinctly different axes of the forefoot. (Fig. 8)
Abduction engages the transverse axis and adduction promotes the oblique axis. The transverse and oblique axes of the forefoot have been described by BojsenMoller. $^{28}$

Abduction or external rotation of the foot results in the predominant action of the transverse axis of the forefoot. The transverse axis consists of the first and second metatarsal heads. The transverse axis lengthens the CT band resistance arm and is employed when high angular velocity of the CT band is the desired moment about the ankle joint. Activities that would benefit from use of the transverse axis include a brisk walk or the initiation of running.

Adduction, or internal rotation of the foot results in the predominant action of the oblique axis of the forefoot. The oblique axis runs between the second and fifth metatarsal heads. The oblique axis shortens the resistance arm and is used when low angular velocity of the CT band is desired. Examples of lower angular velocity would include slow, sustained walking or resisting heavy loads for sustained periods of time.

Table 3. is a summary of the changes in the CT band caused by rotational placement and change in the forefoot axis.

The rotational placement of the foot changes with each step. Carrier speculated that the rotational placement of the foot varied to accommodate and sustain the work of the CT band. ${ }^{29}$ Carrier's theory suggests that the resistance arm length is optimized in response to the ground reactive force (GRF).

Change in rotational placement of the foot is necessary to accommodate different characteristics of human movement. Alteration of walking speed or walking surface will necessitate variations in rotational placement. For instance, Shorten described variation in the rotational position of the resistance arm of the CT band at different walking speeds. ${ }^{30}$ It appears that the rotational placement of the foot is determine by both (1) the need to optimize and minimize work by the CT band and (2) the need to accommodate variations in walking speed or walking surfaces. 
Table 3. Comparison of the forefoot axes formed by rotational placement of the foot.

\begin{tabular}{|l|l|l|}
\hline & Transverse Axis & Oblique Axis \\
\hline Forefoot position & Abduction & Adduction \\
\hline Location of axis & $1^{\text {st }}$ and $2^{\text {nd }}$ metatarsal heads & $\begin{array}{l}2^{\text {nd }} \text { through } 5^{\text {th }} \text { metatarsal } \\
\text { heads }\end{array}$ \\
\hline Effect on forefoot length & Lengthens & Shortens \\
\hline Effect on ankle joint moment & Increases moment & Decreases moment \\
\hline Effect on ankle joint velocity & Increased angular velocity & Decreased angular velocity \\
\hline $\begin{array}{l}\text { Effect on the ground reactive } \\
\text { force (GRF) of the resistance } \\
\text { arm }\end{array}$ & Increases & Decreases \\
\hline Work impact on CT Band & Increases & Decreases \\
\hline Optimal activities & High speed - sprinting & $\begin{array}{l}\text { Slow speed - resisting heavy } \\
\text { loads }\end{array}$ \\
\hline
\end{tabular}

Observation of rotational placement in different activities has been studied and has resulted in contradictory results. ${ }^{31-33}$ Each of these studies has relied upon the definition of walking and running to be the result of active push-off theory as defined by Winter and Kepple. ${ }^{13,33}$ For instance, the transverse axis has been described in Table 3 as the axis that would result in the greatest angular velocity around the ankle and subsequently, higher resistance to GRF. It would stand to reason that the transverse axis would be the optimal axis for sprinters.

Fuchs and Staheli found contradictory evidence noting that many high school sprinters have in-toed gait. $^{34}$ Sprinting is accomplished by placing the body's center of mass far ahead of the center of pressure (COP) of the GRF. Therefore, sprinting cannot be simply described by active push-off theory. Instead, we need to recognize sprinting as a controlled fall that incorporates both the controlled roll-off theory of Sunderland and Skinner ${ }^{11,35}$ and the active push off theory of Winter and Kepple. ${ }^{13,33}$ The role of rotational placement of the foot therefore varies by situation and demand.
The transverse and oblique axes are not mutually exclusive of each other, but rather work together to optimize sustainable CT band biomechanics based upon immediate needs. These conflicting theories make it apparent that additional research is required to clarify this topic.

\section{CT Band Dynamics}

In their book Human Walking, Inman et al stated, "In the complex phenomenon of human walking, a single muscle rarely acts alone; rather, it acts in conjunction with other muscles to achieve the required effect'. ${ }^{25}$

O'Connell stated that "living muscles do not act singly, but in groups, their integrated activity resulted in coordinated movement patterns." ${ }^{36}$ Understanding CT band biomechanics requires an acceptance that the CT band is not made up of independent, isolated structures, but instead consists of many structures working as one unit. The CT band accepts force and load from multiple proximal sources and distributes that force and load to many distal structures using the kinetic and kinematic properties previously described. The functional integration of these kinetic and kinematic properties is called CT band dynamics. 
The primary dynamics of the CT band are the restraint of forward motion of the tibia on the talus and the conservation of work performed by the body by maintaining the body's center of mass upright and within the sagital plane. ${ }^{11}$ Restraint of forward motion of the tibia on the talus is accomplished by eccentric contraction of the CT band. Eccentric contraction of the CT band slows the tibia as it moves forward over the ankle rocker. Eccentric contraction is a more sustainable muscular function, requiring far less work than concentric contraction. ${ }^{4,7}$ As an example, if we look at the function of the biceps muscle, it is much easier for the biceps muscle to lower a weight (eccentric contraction) than it is to raise a weight (concentric contraction). The CT band takes advantage of this biomechanical advantage and conserves energy through sustained, slow eccentric contraction.

The secondary dynamics of The CT Band include stability of the knee, ankle, subtalar joint, midtarsal joint and metatarsal phalangeal joints.

Kinetics, as previously mentioned, is one of the two properties of CT band dynamics. The kinetic properties of the CT band are dictated by skeletal muscle. Skeletal muscle functions as a motor, a spring, a shock absorber and a stabilizer. ${ }^{37}$ In human biomechanics, skeletal muscle normally assumes only one of these functions. The CT band is unique in that it can assume one or several of these functions simultaneously. The CT band functions primarily as a stabilizer, limiting the forward excursion of the tibia. However, eccentric contraction of the CT band may be converted to elastic energy and released as positive work (concentric contraction). This action can be compared to a spring. Hof described this phenomenon which is very pertinent to CT band biomechanics.(38) As the tibia moves forward over the ankle rocker, the CT band is storing energy that will be transferred to the forefoot rocker. It is interesting to note that EMG studies have found that the CT band doesn't actually contribute to active push off at the toe off phase of gait.
At toe off in normal gait, the amount of force delivered by the CT band rapidly diminishes, resulting in no active push off by the CT band. The elastic energy stored in the CT band during the midstance phase of gait is used to maintain a final isometric contraction at the forefoot rocker prior to the toe off phase of gait. This isometric contraction is much like a spring and a shock absorber. In cases where aggressive forward locomotion is needed, the CT band will switch from eccentric to concentric contraction and function as a motor. With this change to concentric contraction, the CT band changes from isometric to isotonic contraction. Therefore, the skeletal muscle in CT band dynamics is unique in that it functions as a motor, a spring, a shock absorber and a stabilizer.

CT Band dynamics are an interesting mixture of kinetic and kinematic properties that enable us to stand upright and walk. Each step represents a unique orchestration of these properties based upon (1) the rate of walking (2) changes in the contact surface or (3) a change in the location of the body's center of mass. Surprisingly there is no theoretical consensus that describes how we walk. A review of the literature finds four main theories that describe how we walk. These theories include:

- Controlled roll-off (Sunderland, Perry and Skinner). ${ }^{11,12,35}$

- Active forward progression as the result of push-off. (Winter and Kepple). ${ }^{13,33}$

- Acceleration into swing phase (Meinders and Hof). ${ }^{14,40}$

- Support of the tibia and trunk to enable interchange of trunk potential and kinetic energy (Neptune and Mochon., ${ }^{1,41}$

Neptune astutely recognized that these theories may not be mutually exclusive.(1) Central to each of these theories of gait is CT band dynamics. 


\section{CT Band Syndrome}

The inability of the CT band to sustain repetitive load may result in injury to one or more of the components of the CT band. These injuries are called CT band syndrome (CTBS). Factors that contribute to CTBS may be physiological, mechanical or a combination of both.

Physiological factors that may contribute to weakening of the CT band include:

- Increased age.

- Weight gain and obesity.

- Malnutrition.

- Poor physical condition.

Mechanical factors that result in increased stress to the tissue structures of the CT band include:

- Increased repetition of load.

- Increased duration of load.

- Increased amount of load.

- Eccentric loading.

CT band syndrome has a translational (CTBS-1) and/or rotational (CTBS-2) basis for its onset, continued presence or recurrence.

CTBS-1 - Translational Conditions and Injuries

Injuries that are the result of sagital plane, longitudinal strain within the CT band. Common CTBS-1 conditions would include plantar fasciitis, Achilles tendonitis and Sever's disease.

CTBS-2 - Rotational Conditions and Injuries -

CTBS-2a - The cause of rotation.

Intrinsic conditions or deformities that contribute to rotation and shear strain within the CT band. Examples include PTTD, metatarsus adductus, calcaneal varus, calcaneal valgus, etc.

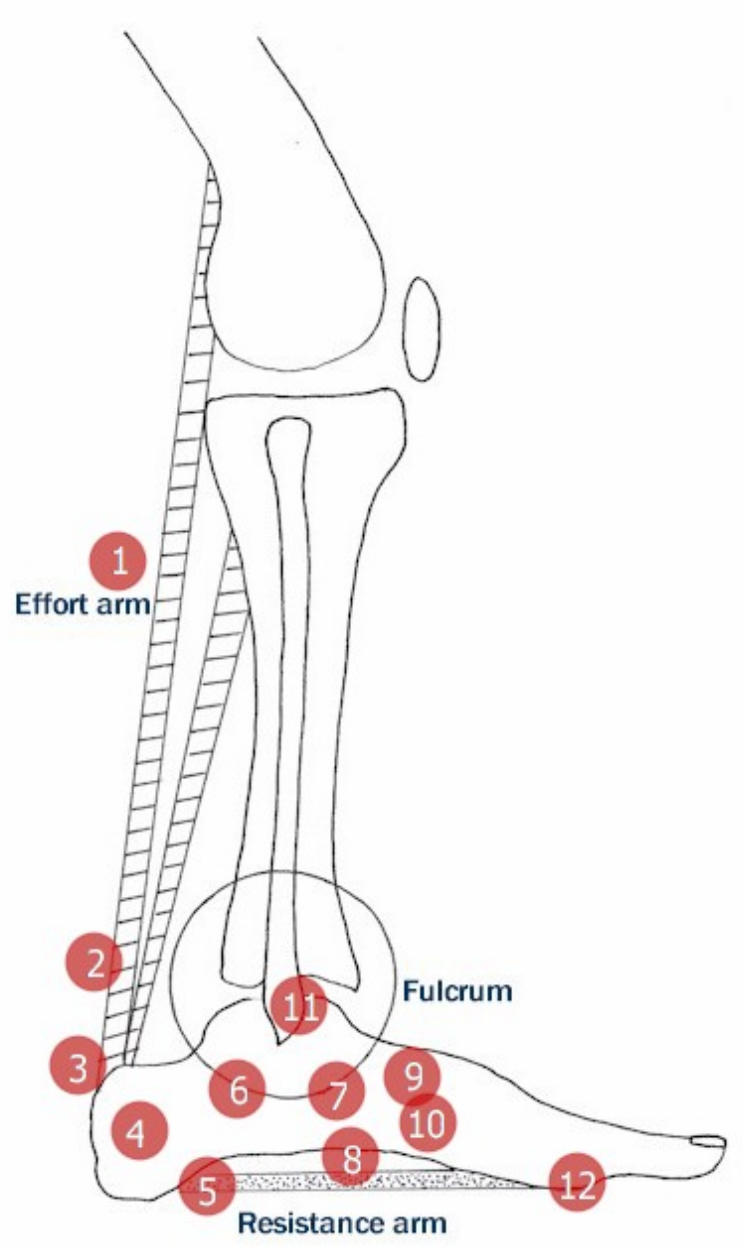

Figure 9 CT band syndrome- common locations of pathology along the CT band: (1) calf cramping and stiffness secondary to equinus, (2) chronic Achilles tendonitis and micro tears of the Achilles tendon, (3) insertional Achilles tendonitis, (4) Sever's Disease, (5) plantar fasciitis, (6) tarsitis, (7) posterior tibial tendon dysfunction, (8) peroneal tendonitis, (9) Lisfranc's joint arthritis, (10) Charcot joints, (11) forefoot bursitis, capsulitis and ulcerations, (12) flat top talus and chronic ankle pain.

CTBS-2b - The effect of rotation.

Injuries that are the result of a causal relationship that is dependent upon (a) the presence of CTBS-2a and (b) the addition or input of a stressor that has a negative effect on pre-existing CTBS-1. Stressors may be physiological or mechanical. 


\section{Stress/strain, stress risers and tissue elasticity}

When load is applied to the CT band, tissue within the band will undergo stress. Sustained CT band function requires ongoing physiological management of stress. CT band syndrome occurs when the CT band is unable to repair accumulated stress resulting in strain of soft tissue or bone.

Strain is the geometric change in soft tissue and bone that signifies injury. There are two types of strain: longitudinal and shear strain. CTBS-1 injuries are the result of longitudinal strain. Longitudinal strain within the CT band results in the lengthening and subsequent thinning of tendon, muscle and ligaments. As longitudinal stress is applied to a tissue, the cross sectional area of the tissue perpendicular to the stress

Tissue elasticity and its' relationship to injury and healing is a significant variable to be considered in CTBS. In addition to the primary musculo-tendinous structures of the CT band, force is carried by numerous other structures including ligaments, joints and bone. The elasticity of these structures is essential to sustained CT band function. These structures participate in CT band dynamics by resisting, transmitting and absorbing force. Tissue elasticity is the ability of tissue to accommodate stress and is known to decrease with age. Therefore, we can assume that CT band syndrome will be more prevalent in aging, yet active patients.

\section{CT Band Syndrome as a repetitive use injury}

Repetition in CT band dynamics may be a contributing cause of CT band syndrome. In existing CTBS-1 and CTBS-2b, failure to address CT band dynamics may be considered a factor that perpetuates the injury. Therefore, CTBS can be considered a form of repetitive use syndrome. decreases, resulting in strain. This relationship of tissue deformation is known as Poisson's Effect. ${ }^{42}$

CTBS-2a deformities promote uncompensated rotation of the CT band. As load, force or other stressors are applied to the CTBS-2a deformity, shear strain develops resulting in CTBS-2b injuries.

Stress risers within the CT band are a contributing cause of CT band syndrome. Stress risers are focal areas where stress is potentiated with applied load or force. Stress risers absorb force and limit the effective transfer of force from the effort arm to the distal aspect of the resistance arm of the CT band. Examples of stress risers within the CT band may include but are not limited to arthritis, tendon injuries and stress fractures. Structural deformities of the foot (CTBS 2-a) can contribute to rotation and become inherent stress risers with repetitive use.

\section{Treating CT Band Syndrome}

\section{Sagittal Plane CT Band Syndrome (CTBS-1)}

CTBS-1 occurs as the result of longitudinal strain in the sagittal plane. CTBS-1 can be treated by weakening the CT band in the sagital plane. Weakening the CT band in the sagittal plane is accomplished by the following methods:

Shoe Modifications

- Heel lifts.

- Wedge sole shoes.

- Forefoot and rearfoot rocker shoe modifications.

Physical Therapy

- Calf stretching.

Surgery

- Gastrocnemius recession.

- Tendo-Achilles lengthening. 


\section{Frontal and Transverse Plane CT Band Syndrome (CTBS-2)}

Treatment of CTBS-2 necessitates realignment of the CT band in an attempt to decrease rotation and maximize delivery of translation in the sagittal plane. Methods used to treat CTBS- 2 include:

Shoe Modifications

- Rigid shank.

- Laced leather uppers on shoes.

- Stiff leather sole shoes.

- Leather Oxfords Thomas heels/reverse Thomas heels.

- Sole wedges.

- Lateral and medial flair.

Foot/Ankle Bracing

- Rigid AFO/SMO.

- Dynamic AFO/SMO.

- Foot orthoses.

Surgery

- Metatarsus adductus correction.

- Medial or lateral column shortening or lengthening.

- Pes planus correction.

- Pes cavus correction.

- Tendon balancing procedures.

- Triple arthrodesis with or without wedging.

- Calcaneal displacement osteotomy.

- Subtalar arthroeresis.

In conclusion, lower extremity biomechanics has traditionally been taught in a way that we learn to look at function as a single muscle and pathology as an isolated problem. Defining the CT band and CT band syndrome enables providers to view lower extremity biomechanics based upon lever mechanics.
The CT band is an example of a lever where "living muscles do not act singly, but in groups, their integrated activity resulted in coordinated movement patterns." 43 This integration of anatomy, kinetics and kinematics is called CT band dynamics.

CT band syndrome is described as a group of related conditions and injuries that share as their common denominator, the CT band. Factors contributing to CTBS may be mechanical, physiological or a combination of both. CTBS is often an overuse syndrome common to the aging yet active patient population. CTBS can be predominantly translational (CTBS-1), rotational (CTBS-2) or a combination of both. CTBS-1 is a translational injury that occurs due to longitudinal strain within the sagital plane. CTBS-2 describes shear strain injuries that result from rotation. CTBS-2a represents the intrinsic cause of ration while CTBS2-b describes the shear strain injury due to rotation.

Acknowledgments - The author wishes to thank Michael Forman, DPM, Cleveland, Ohio for his critical review of this paper. The author would also like to thank Thalia Oster for her support, encouragement and editorial focus.

\section{References}

1. Neptune RR, Kautz SA, Zajac FE. Contributions of the individual ankle plantar flexors to support, forward progression and swing initiation during walking. J Biomechanics 2001; 34:1387-1398

2. Root ML, Orien WP and Weed JH. Normal and Abnormal Function Of The Foot. Los Angeles: Clinical Biomechanics Corp; 1977

3. Spoor CW, van Leeuwen JL, Meskers CG, Titulauer AF, Huson A. Estimation of instantaneous moment arms of lowerleg muscles. J Biomechanics 1990; 27:1247-1259

4. Asmusen E. Positive and negative muscle work. Acta Physiol Scan 1953; 28:365-382

5. McCully KK, Faulkner JA. Characteristics of lengthening contractions associated with injury to skeletal muscle fibers. J Appl Physiol 1986; 61:293-299

6. Hughston JC, Walsh WM, Puddu G. Patellar tendon subluxation and dislocation. Philadelphia: WB Saunders 1983; 5:1-191

7. Sapega A A. Muscle performance evaluation in orthopedic practice. J Bone Joint Surg 1990; 72-A: 1562-1574 
8. Singh $\mathrm{M}$, Karpovich PV. Isotonic and isometric forces of forearm flexors and extensors. J Appl Physiol 1966; 21:14351437

9. Abbott BC, Bigland B, Ritchie JM. The physiological cost of negative work. J Physiol 1952; 117:380-390

10. Bigland B, Lippold OCJ. The relation between force, velocity and integrated electrical activity in human muscles. J Physiol 1954; 123:214-224

11. Sutherland DH, Cooper L, Daniel D. The role of ankle plantar flexors in normal walking. J Bone Joint Surg 1980; 42-A: 354-363

12. Perry J. Gait Analysis: Normal and Pathological Function. Thorofare, NJ: Slack Inc; 1992.

13. Winter DA. Energy generation and absorption at the ankle and knee during fast, natural and slow cadences. Clinical Orthopedics 1983; 175: 147-154

14. Meinders M, Gitter A, Czerniecki JM. The role of ankle plantar flexor muscle work during walking. Scandinavian Journal of Rehabilitation Medicine 1998; 30:39-46

15. Friederich JA, Brand RA, Muscle fiber architecture in the human lower limb. J Biomech 1990; 23:91-95

16. Wickiewicz TL, Roy RR, Posell PL, and Edgerton, VR. Muscle architecture in the human lower limb. Clin Orthop Rel Res. 1983; 179:275-283

17. Robertson DG, Winter DA. Mechanical energy generation, absorption and transfer amongst segments during walking. J of Biomechanics. 1980; 13:845-854

18. Weber EF. Ueber die Langenverhaltnisse der Fleischfasern der Muskeln im allgemeinen. Berichte uber die Verhandlungen der Koniglich Sachsischen Akademie der Wissenschaften zu Leipsig, Mathematisch-physische Classe. Leipzig: Weidmannsche Buchhandlung, 1851

19. Jergensen FH. Studies in Various factors influencing internal fixation as a method of treatment of fractures of long bones. Report to National Research Council Committee on Veterans Medical Problems, Contract number V1001M-1945, Jan. 5, 1950-July 1, 1951.

20. Haxton HA. Absolute muscle force in the ankle flexors of man. J. Physiol. 1944; 103:267-273

21. Kabada MP, Ramakrishnan HK, Wootten ME.

Measurement of lower extremity kinematics during level walking. Journal of Orthopaedics Research 1990; 8:383-392 22. Novacheck TF. The biomechanics of running. Gait and Posture 1998; 7:77-95

23. Stokes VP, Andersson C, Forssberg H. Rotational and translational movement features of the pelvis and thorax during adult human locomotion. Journal of Biomechanics 1989; 22:43-50

24. Cornwall MW, McPoil TG. Reliability and validity of center of pressure quantification. J Am Podiatr Med Assoc 2003; 93:42-149

25. Inman et al: Human Walking, Williams and Wilkens, Baltimore 1981.
26. Simon SR, Mann RA, Hagy JL, Larsen LJ. Role Of The Posterior Calf Muscles In Normal Locomotion. J Bone J Surg 60A, 465-472, 1978.

27. Erdemir A, Piazza SJ. Rotational foot placement specifies the lever arm of the ground reaction force during push-off phase of walking initiation. Gait and Posture 2002; 15;212219

28. Bojsen-Moller F. The human foot - a two speed construction. In:Asmussen E, Jorgensen K, editors.

Biomechanics V. Baltimore: University Park Press 1978; 2616

29. Carrier DR, Heglund NC, Earls KD. Variable gear during locomotion in the human musculoskeletal system. Science 1994; 265:651-3

30. Shorten MR, Eden KB, Himmelsbach JA. Plantar

pressures during barefoot walking, XII International Congress of Biomechanics, Los Angeles, 1989.

31. Rosenbaum D, Hautmann S Gold M, Claes L. Effects of walking speed on plantar pressure patterns and hindfoot angular motion. Gait Posture 1994; 2:191-7

32. Ho C-S, Lin C-J, Chou Y-L, Su F-C, Lin S-C. Foot progression angle and ankle joint complexes in preschool children. Clin Biomechanics 2000;15:271-7

33. Kepple TM, Siegel KL, Stanhope SJ. Relative contributions of the lower extremity joint movements to forward progression and support during stance. Gait and Posture 1997; 6: 1-8

34. Fuchs R, Staheli LT. Sprinting and intoeing. J Pediatric Orthop 1996;16:489-91.

35. Skinner SR, Antonelli D, Perry J, Lester DK. Orthopedics 1985; 8: 355-361

36. O’Connell AL. Electromyographic Study Of Certain Leg Muscles During Movements Of The Free Foot And During Standing.

37. Stauber WT. Eccentric action of Muscles. Exercise and sport science reviews 17 157-185, 1989.

38. Hof AL, Geelen BA, Van den Berg JW. Calf Muscle Moment, Work and Efficiency In Level Walking; Role of Serial Elasticity. J Biomechanics 16:523-537, 1983.

39. Skinner SR, Antonelli D, Perry J, Lester DK. Orthopedics 1985; 8: 355-361

40. Hof AL, Nauta J, Vanderknaap ER, Schallig MAA, Struwe DP. Calf muscle work and segement energy changes in human treadmill walking. Journal of Electromyography and Kinesiology 1993; 2: 203-216

41. Mochon S, McMahon TA. Ballistic Walking. Journal of Biomechanics 1980; 13:49-57

42. Frankel VH, Burstein AH. Orthopedic Biomechanics. Philadelphia, Lea \& Febiger, 1970.

43. Siegler S, Moskowitz GD, and Freedman W. Passive and Active Components of the Internal Moment Developed about the Ankle Joint During Human Ambulation. Journal of Biomechanics 1984; 17: 647-652 